\title{
Basal transcription machinery: role in regulation of stress response in eukaryotes
}

\author{
*Parag Sadhale, Jiyoti Verma and Aruna Naorem \\ Department of Microbiology and Cell biology, Indian Institute of Science, Bangalore 560 012, India \\ *Corresponding author (Email,pps@mcbl.iisc.ernet.in)
}

\begin{abstract}
The holoenzyme of prokaryotic RNA polymerase consists of the core enzyme, made of two $\alpha, \beta, \beta$ ' and $\omega$ subunits, which lacks promoter selectivity and a sigma $(\sigma)$ subunit which enables the core enzyme to initiate transcription in a promoter dependent fashion. A stress sigma factor $\sigma^{s}$, in prokaryotes seems to regulate several stress response genes in conjunction with other stress specific regulators. Since the basic principles of transcription are conserved from simple bacteria to multicellular complex organisms, an obvious question is: what is the identity of a counterpart of $\sigma^{s}$, that is closest to the core polymerase and that dictates transcription of stress regulated genes in general? In this review, we discuss the logic behind the suggestion that like in prokaryotes, eukaryotes also have a common functional unit in the transcription machinery through which the stress specific transcription factors regulate rapid and highly controlled induction of gene expression associated with generalized stress response and point to some candidates that would fit the bill of the eukaryotic $\sigma^{s}$.
\end{abstract}

[Sadhale P, Verma J and Naorem A 2007 Basal transcription machinery: role in regulation of stress reponse in eukaryotes; J. Biosci. 32 569-578]

\section{Stress response}

Stress can be broadly defined as any unfavourable condition. A given condition may or may not be stressful to an organism hence the stress response elicited by a given condition is dependent on the organism as well as the stressor. The stresses in general can be categorized into different groups as enlisted in table1. The physical/chemical parameters like high/low temperatures, $\mathrm{pH}$, presence of toxic metal ions, osmolarity, and water content of the growth medium etc. are perceived as stress conditions by a variety of organisms. In fact, cells even respond to mechanical stress as sensed by the cell membranes/cell walls exerted internally by the turgor pressure or externally by increased atmospheric pressure. In cardiac muscle cells the differential activation of PKC (protein kinase C) pathways leads to activation of transcription factors causing differential gene expression in response to the mechanical stress perceived by the muscle cells. These differentially expressed genes have been associated with cardio myopathies and thus are of great significance (Hoshijima 2006). Even the simple bacterial cell needs to maintain the integrity of its cell envelope for survival, the trans-membrane signal-transducing protein factors monitor its perturbations and respond appropriately by modulating gene expression (Wecke et al 2006). While there is a large variety of stresses that living systems respond to, the most prevalent and common stress condition in nature is starvation, i.e. limiting of one or more nutrients.

Whereas, the response to heat shock as stress has been studied in great detail in a variety of systems and found to be highly conserved with respect to the heat shock proteins induced, the stresses like starvation and other environmental stressors, like competing microflora or invading pathogenic organisms, elicit complex and varied responses dependent on the organism in question. Interestingly, it has been noted in several organisms that when an organism responds to one stress, it often shows increased ability to cope with other stresses and indeed a particular stress is able to cause induction of genes required to function in response to an unrelated stress. This may be well justified from an evolutionary perspective, since in nature stresses are not encountered in isolation. Typically a cell which encounters

Keywords. Basal transcription; eukaryotes; stress response 
Table 1. Broad categories of cellular stress

\begin{tabular}{ll}
\hline Type of stress & Parameters \\
\hline Physical/chemical & $\begin{array}{l}\text { Temperature, } \mathrm{pH} \text {, presence of toxic metal } \\
\text { ions, Osmolarity, Water level }\end{array}$ \\
Mechanical & $\begin{array}{l}\text { Atmospheric or turgor pressure leading to } \\
\text { mechanical stress on membranes }\end{array}$ \\
Starvation & Absence of one or more nutrients \\
\hline
\end{tabular}

one stress is likely to encounter another stress. Hence, the chance of survival might be higher if the given cell encountering a stress is prepared to cope with more than just that stress at the same time. Mechanistically, it might mean that the regulation of stress response may be linked and once the mechanism is activated by one stress, the cell might become predisposed to respond to another stress. This is indeed observed in nature and illustrated in several examples listed below.

Streptomyces coelicolor undergoing sporogenic differentiation in liquid culture also exhibits higher resistance to oxidative damage. In this case as well, a correlation between the starvation stress and the oxidative stress and their regulators has been proposed (Lee et al 2005). HOG1 gene in Saccharomyces cerevisiae encodes a MAP kinase that controls the hyper-osmotic stress response. The homolog of HOG1, in Trichoderma harzianum, a widespread mycoparasitic fungus, when silenced, shows intermediate levels of resistance against oxidative stress (Delgado-Jarana et al 2006). It has been proposed that the increased susceptibility of proteins to oxidative damage may be due to some damage to the cellular components simultaneously leading to increased chaperone levels. In Escherichia coli, nutritional downshift in stationary phase is associated with greater tolerance to the oxidative damage (Dukan and Nystrom 1999). In mammalian kidney cells, heat shock and osmotic shock elicit overlapping response (Santos et al 1998). It is of course possible that some common signal(s) or intracellular phenomena are shared by osmotic and growth-limiting (associated with the stationary phase or starvation condition) and/or other stress conditions. However, an interesting alternative emerging from the recent studies is the presence of shared regulatory cascades which might result in overlapping responses by each of these apparently distinct stress conditions.

While instantaneous, or rapid response to a stress may appear to be best achieved at the level of activation of functional molecules like proteins, to have the protein molecules required for a stress specific function synthesized before stress condition is encountered, is not likely to be economical or efficient. So, the cells resort to mechanisms which would enable them to synthesize the rescue operators rapidly on sensing stress. Most often this is achieved by regulating the synthesis of the required proteins at the transcriptional level. We will discuss in this review, in brief, the different factors associated with the basal transcription machinery that might contribute to the regulation of gene expression at the transcriptional level in response to a wide variety of stresses. Conceptually, a greater control over wider spectrum of genes could be achieved if the regulator is close to the core of the transcription machinery and it would enable the system to rapidly and simultaneously respond to a number of stresses at the transcriptional level.

The process of transcription is highly regulated at the level of transcription initiation. The differential transcription initiation is brought about by altered promoter selectivity by the transcription machinery. In prokaryotes, the sigma subunit of the RNA polymerase dictates specificity towards the promoter. The gene specific transcription factors and other ancillary factors further fine tune the level and specificity of expression. Extensive studies have been carried out to elucidate the mechanism of how gene specific transcription factors function both in prokaryotes and in eukaryotes, but the role of the basal transcription machinery in transcriptional regulation in stress is well studied mainly in prokaryotes hence we first briefly discuss how the prokaryotic transcription machinery regulates transcription of genes involved in stress response and then seek parallels in the complex eukaryotic system.

\section{Prokaryotic transcription and role of sigma factors in stress response}

In E. coli, the core RNA polymerase consists of five different proteins, namely two of $\alpha$ subunits, one each of $\beta, \beta^{\prime}$ and $\omega$ subunits. RNA polymerase transcribes genes from a specific region of the gene called promoter. In prokaryotes, the sequences at -10 and -35 regions in the promoter are important for polymerase binding for transcription. At the initiation of transcription, the holoenzyme is formed by the association of core polymerase with $\sigma$ factor which helps in the promoter selection. The different sub domains of the DNA binding domain of the sigma factor contact the different regions of the conserved promoter (figure 1). Structure determination has revealed that the $\sigma$ protein in the holoenzyme is in a stretched conformation which enables it to interact with these parts of the promoter (Vassylyev et al 2002). After the promoter recognition, the sigma factor dissociates from the core polymerase and mRNA synthesis is carried out by the core polymerase. Transcription of a gene can be regulated by the modification of core polymerase subunits, interaction with the regulatory proteins or changes in the regulatory regions of the gene. In addition to the above known modifications, studies have shown that mechanism underlying the global regulation of genes is achieved by different sigma factors alternatively associating with the core RNA polymerase. 

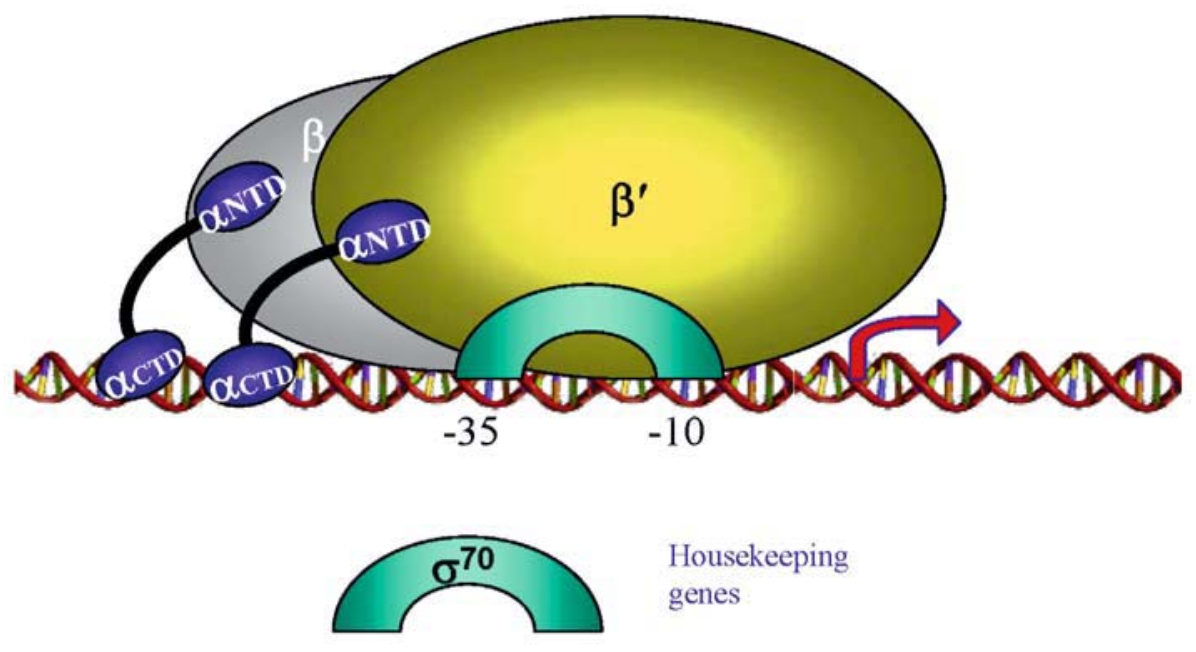

Housekeeping

genes

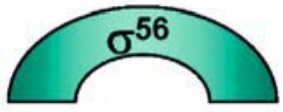

Nitrogen-regulated

genes

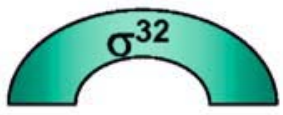

Heat shock

genes

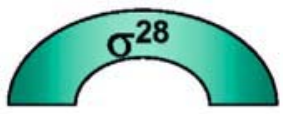

Flagella-chemotaxis

genes

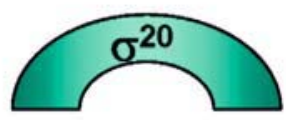

Extreme heat-shock

genes

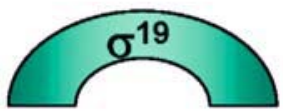

Ion-transport

Figure 1. Prokaryotic core transcription machinery recognizes the promoter which has -10 and -35 elements. The extended conformation of the $\sigma$ factor binds to these elements of the basal promoter. The N-terminal domain of each of the two $\alpha$ subunits, binds to the core polymerase while the $\mathrm{C}$ - terminal domain interacts with the upstream promoter elements. The five alternative sigma factors in $E$. coli are shown along with the genes they regulate.

To explain the role played by sigma factors in protecting the prokaryotic cell from any kind of unfavorable conditions, we shall take up the most extensively studied prokaryote Escherichia coli and a few other bacteria. There are at least six different sigma factors in E. coli identified so far which are known to associate with the core polymerase under different conditions to transcribe specific sets of genes (figure 1). The ratio of the major and alternate sigma factors determines which genes get transcribed at a given time. The major and alternative sigma factors have two distinct conserved domains which are required for binding to the core enzyme and to the basal promoter (Gruber and Gross 2003). In spite of the differences at the protein sequence level, these sigma factors show remarkable structural conservation between these domains. The differences in their abilities to compete with the housekeeping sigma factor and the promoter elements probably stem from the minor sequence variations in these domains. The choice of genes regulated by different sigma factors can be attributed to the differences in the sequences around the -10 and -35 regions and also to the number of bases separating these two sequence elements. In fact the promoter binding domain 4 of each of these $\sigma$ factors interacts differentially with the extended -10 and -35 regions of the respective promoter and allows the strength of binding between the promoter and the $\sigma$ factor to be altered. Promoter sequences at -10 and -35 regions recognized by these alternate sigma factors are not well known except for a few sigma factors. The $\sigma^{70}$ recognizes most of the promoters which are transcribed during exponential phase of growth. A novel sigma designated as $\sigma^{32}$ was isolated along with $\sigma^{70}$ from heat stressed $E$. coli which was later found to regulate a subset of genes encoding proteins commonly called heat 
shock proteins (Grossman et al 1984). These heat shock proteins have a wide variety of functions essential for survival of the organism during a variety of stress conditions (Ishihama 1990). Global transcriptional studies also show that $25 \%$ of $\sigma^{32}$ regulon members are found to be present at the membrane after heat stress suggesting their involvement in protecting the cell membrane in response to the stress (Nonaka et al 2006).

Several stress responses are interlinked by alternative sigma factors which help RNA polymerase to choose promoters of stress genes. The $\sigma^{32}$ is activated by cytoplasmic stress while $\sigma^{\mathrm{E}}$ is activated by extracytoplasmic stress. These stresses, resulting from accumulation of mis-folded or immature proteins in the cytoplasm or the cell envelope, will change the active pool of these sigma factors which are otherwise kept inactive by anti-sigma factors. The third alternative sigma factor, $\sigma^{\mathrm{s}}$ controls a set of genes required during stationary phase. There seems to be considerable amount of overlap in the induced genes during hyperosmotic, and low $\mathrm{pH}$ stress with $\sigma^{\mathrm{S}}$-dependent genes (Bearson et al 1996; Muffler et al 1996). Besides performing their unique tasks in handling unique stresses, these sigma factors co-operate to respond to hyperosmotic stress. The ultimate result of the concerted effort leads to adaptation by which $E$. coli survives a variety of adverse growth conditions (Bianchi and Barney 1999).

The general stress response sigma factor enables the cells to respond to the growth-limiting stresses and also protects the organism from any further stress. $\sigma^{\mathrm{S}}$ of $E$. coli and $\sigma^{\mathrm{B}}$ of Bacillus subtilis and other gram positive species are among the well studied general stress response sigma factors. $\sigma^{\mathrm{B}}$ regulates over 200 genes in response to stresses like heat, oxidative conditions, acidic $\mathrm{pH}$, salt etc (Wecke et al 2006). Its homologs in various species of bacteria regulate virulence, adherent biofilm formation in response to varied stress stimuli. The role of B. subtilis $\sigma^{\mathrm{B}}$ in cell envelope response is well understood. The integrity of cell wall or envelope of bacteria is crucial for survival of the organism as they encounter many unfavorable chemicals in normal habitat, for example, soil, intestines etc. Studies using Bacillus licheniformis, a close relative of B. subtilis, have revealed that even though there is conservation of sigma factors, presence or absence of additional trans-acting proteins makes sigma factor respond differently to the cell envelope stress. Unlike B. subtilis, $\sigma^{\mathrm{B}}$ dependent promoter activation is not seen even in the presence of functional $B$. licheniformis $\sigma^{\mathrm{B}}$ homolog under cell envelope stress (Wecke et al 2006).

Study of the functional homolog of $E$. coli $\sigma^{\mathrm{S}}, \sigma^{\mathrm{B}}$ in Streptomyces coelicolor, has shown that $\sigma^{\mathrm{B}}$ induces a wide variety of defense proteins, sigma factors to overcome the osmotic shock as well as oxidative stress. It induces itself and also its two paralogs, which work in cascade to ensure proper and efficient sporulation of $S$. coelicolor. Thus $\sigma^{\mathrm{B}}$ plays an important role in maintaining proper differentiation of the organism and to counter different stress conditions (Lee et al 2005). In some of the gram positive pathogenic organisms like Listeria monocytogenes, and S. aureus, pathogenesis appears to be tightly associated with stress response and several virulence factor genes are controlled by the stress sigma factor $\sigma^{\mathrm{B}}$ (Schaik and Abee 2005). Thus, the mechanistic link in regulating responses to apparently unrelated stresses as well as conditions conducive for expression of virulence factors etc., in prokaryotes, lies in the shared regulators which are part of the basal transcription machinery.

\section{Eukaryotic transcription}

Unlike prokaryotes, the process of transcription in eukaryotes is much more complex in keeping with the fact that transcriptional machinery has to function with the larger genomes that are packaged into higher order chromatin structure. In addition, in most multi-cellular organisms, the temporal, spatial and tissue specific regulation of gene expression is crucial. All these factors contribute to the increase in complexity of the transcription machinery. Although eukaryotic transcriptional machinery consists of a larger number of protein complexes than that of prokaryotes, the general principles of transcription and its regulation are conserved. The task of eukaryotic transcription is shared by three different RNA polymerases I, II and III, which synthesize different classes of RNA. Among these the RNA polymerase II (Pol II), which catalyzes the transcription of all protein coding genes, has been studied in greater detail. In yeast as well as humans, the pol II is composed of 12 subunits, designated Rpb1 to Rpb12. Several of these subunits (Rpb5, Rpb6, Rpb8, Rpb10 and Rpb12) are shared with the other two polymerases. In addition, Rpb1, $\mathrm{Rpb} 2, \mathrm{Rpb3/Rpb11}$ and Rpb6 are homologous to bacterial core RNA polymerase subunits $\beta^{\prime}, \beta, \alpha$ and $\omega$ respectively (Hampsey 1998). Rpb9 is important in active site selection (Hampsey 1998) and recently it has also shown to play a role in transcription coupled repair. Rpb4 and $\mathrm{Rpb} 7$ form a separate sub-complex of the pol II in S. cerevisiae that has been shown to have a variety of roles (see below).

A large number of transcription factors and several protein complexes assist the polymerase in its function. Six general transcription factors, TFIIA, TFIIB, TFIID, TFIIE, TFIIF and TFIIH are important for accurate initiation of transcription by RNA polymerase (reviewed in detail by Thomas and Chang 2006). TFIID is one of the primary factors which recruits on promoter and helps in further assembly of other general transcription factors and pol II to form pre-initiation complex (PIC). This multi-subunit complex recognizes several elements of eukaryotic promoter (figure 2): the 


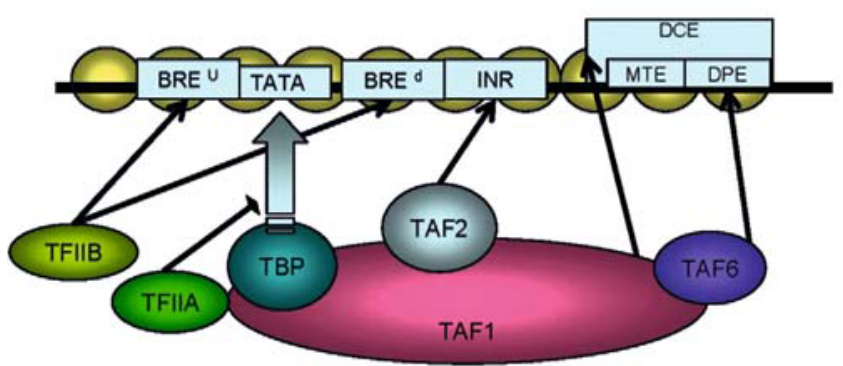

Figure 2. The eukaryotic promoter is much more complex with several different elements, each of which interacts with a different component of the core transcription machinery. The components of the TFIID complex, the TBP and the TBP associated factors (TAFs) interact with the TATA element and with various other elements in the basal promoter respectively. TFIIB interacts with the $\mathrm{B}$ responsive elements flanking the TATA box while TFIIA affects the TBP interaction with the TATA element (indicated by a blunt arrow). This suggests that unlike the single sigma factor in the prokaryotic polymerase, several protein factors in the basal transcription machinery interact with the basal promoter in the eukaryotes. Only the factors known to interact with the promoter elements or known to affect directly the interactions of other factors are depicted.

TATA box, an A/T rich sequence present approximately 25 to 30 nucleotides upstream of transcription start site, is recognized by TATA binding protein (TBP); initiator (INR), a pyrimidine rich sequence, is recognized by TAF1/TAF2 subunits of TFIID, and promoter element called downstream promoter element (DPE), is recognized by TAF6 and TAF9 subunits of human TFIID (Shao et al 2005). Another transcription factor which makes contact with the promoter is TFIIB which recognizes two TFIIB-recognition elements (BRE) present upstream $\left(\mathrm{BRE}^{\mathrm{u}}\right)$ and downstream $\left(\mathrm{BRE}^{\mathrm{d}}\right)$ of TATA box (figure 2). In addition to these elements, another consensus sequence is present between +18 to +29 , called MTE (Motif Ten Element) which is shown to enhance PolII mediated transcription in conjunction with INR (Lim et al 2004) but its cognate protein factor is not known.

Besides these general transcription factors, several cofactors are required for transcription initiation. These cofactors can be divided into two classes. The first class includes factors or enzymes required for modification of chromatin. The second class contains factors which are important for interaction with RNA pol II and general transcription factors. The proteins belonging to this class are collectively called mediator complex because they serve as a bridge between activator and the basal transcriptional machinery (Chadick and Asturias 2005). Mediator complex is composed of $\sim 21$ subunits which can be divided in four sub-modules. The head module, composed of Med6, Med8, Med11, Med17 (Srb4), Med18 (Srb5), Med19 (Rox3),
Med20 (Srb2), and Med22 (Srb6), is thought to have a general role in transcription and interacts with the CTD of RNA pol II (Lee and Kim 1998). The second module, the middle domain, which is composed of Med1, Med4, Med5 (Nut1), Med7, Med9, Med10, Med14 (Rgr1), and Med21 (Srb7), interacts with the CTD of RNA pol II similar to the head module. The subunits of the tail module, composed of Med2, Med3 (pgd1), Med15 (Gal11), and Med16 (Sin4), have been identified by genetic methods. This module is presumably responsible for recognizing and binding to activators (Bhoite et al 2001). A fourth distinct sub-complex (also called Cdk8 sub-complex) that includes mediator subunits Cdk8 (Srb10), CycC (Srb11), Med12 (Srb8), and Med13 (Srb9) has been implicated in negative regulation of transcription (Hampsey 1998). A kinase defective mutant of Cdk8 showed, on genome wide transcription profiling, up-regulation of significant subset of genes (Holstege et al 1998). Recent studies have shown that the Ras/PKA pathway can modulate mediator activity (Chang et al 2004) suggesting that mediator can have direct signalingprocessor role.

\subsection{Does the eukaryotic transcription machinery have sigma analogs involved in stress response?}

From the above discussion it is clear that there is no single protein in eukaryotes that functions in identical manner as the $\sigma$ subunit of the prokaryotic polymerase, in that a protein enables the polymerase to interact directly with the specific sequence on the DNA. Considering the complexity of the transcription machinery in the eukaryotes one could envision that the functionality of the $\sigma^{70}$ factor is taken over by a complex of proteins e.g. TFIID, a complex made up of TBP and its associated factors (TAFs), which allows the association of the polymerase with the promoters of most of the housekeeping genes. The gene specific transcription factors that regulate activities of specific genes or subsets of the TFIID controlled genes can function over and above the TFIID. This is similar to several gene specific transcription regulators that function in prokaryotes in conjunction with the sigma factors in modulating specific gene transcription. What follows the above argument is that if we look for factors which can function similar to $\sigma^{\mathrm{S}}$ subunit during stress response, three factors in eukaryotic transcription machinery, discussed below, come to the fore.

3.1.1 SAGA complex: The SAGA complex, SPT3-ADA2GCN4-histone Acetyltransferase is known to contain the TBP and some of the TBP associated factors along with other components that enable the complex to take part in histone acetylation required for chromatin remodeling. This 1.8MDa multi-subunit complex comprises of many distinct classes of proteins: (i) the Ada proteins (Ada1, 
Ada2, Ada3, Gen5 and Ada5); (ii) Spt proteins (Spt3, Spt7, Spt8 and Spt20); (iii) a subset of TBP-associated factors (TAFs: Taf5, Taf6, Taf9, Taf10 and Taf12; and (iv) Tra1, an ataxia telangiectasia mutated (ATM)-related protein, which plays an important role in activator recruitment (Brown et al 2001). Several components of the TFIID complex are also part of this complex. Gcn5 is the catalytic histone acetyltransferase (HAT) subunit of the SAGA complex but this activity is not always required for all gene activation events regulated by this complex. SAGA complex is extensively studied in $S$. cerevisiae and most of the knowledge about its structure, function and regulation has come from this model system. The primary function of this complex is to help in the delivery of TBP onto promoters. Moreover, SAGA also acts as an adapter in making contacts with other complexes of transcription machinery (Larschan and Winston 2001; Barbaric et al 2003) to form proper PIC similar to $\sigma$ factor in bacteria. Higher organisms also have complexes similar to SAGA complex, e.g., TFTC (TBP free
TAF- containing complex), PCAF (p300 and CBP associated factor) and STAGA are human homologs of SAGA complex (Brand et al 1999; Ogryzko et al 1998; Martinez et al 1998; Martinez et al 2001). However, detailed information about their role in these organisms is lacking. Studies done in $S$. cerevisiae have shown that SAGA complex is mainly associated with promoters which contain TATA box consensus sequence $(\mathrm{A} / \mathrm{T}) \mathrm{A}(\mathrm{A} / \mathrm{T})(\mathrm{A} / \mathrm{G})$ (Basehoar et al 2004). Furthermore it has been shown that the SAGA complex regulates approximately $10 \%$ of the genes in the yeast genome (Huisinga and Pugh; 2004). These genes are highly induced by a variety of environmental stresses such as heat, starvation etc. In another study, this group, using chromatin immuno-precipitation (ChIP) assay conducted on genome-wide scale (ChIP on chip), has shown that the SAGA complex assembly occurs on the promoters which are activated during heat stress in response to heat shock (Zanton and Pugh; 2004). Their study also suggests that SAGA assembly always correlates with gene activation. A SAGA
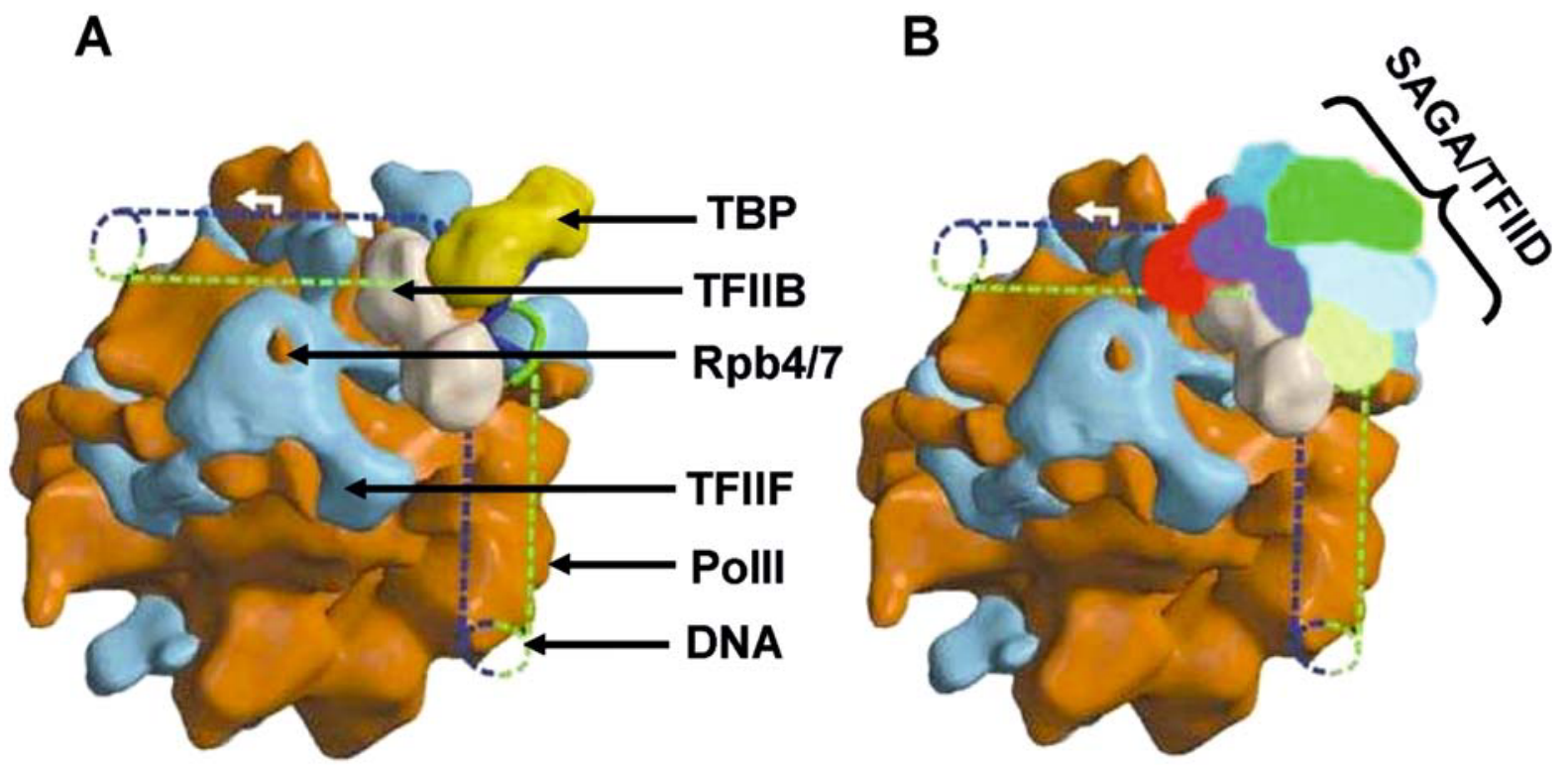

Figure 3. (A) Model of the structure of a minimal transcription complex. RNAPII-IIF-IIB-TBP and DNA constitute the minimal complex that is capable of promoter-directed initiation, and therefore the catalytic core of the eukaryotic transcription machinery. The trajectory of DNA along the RNAPII surface (predicted using the distribution of IIF density in the RNAPII-IIF complex), along with the TATA box to transcription start site spacing of a typical eukaryotic promoter, the expected location of IIB on the surface of RNAPII and the X-ray structure of the TBP-IIB-DNA complex lead to a model for the organization of the catalytic core of the eukaryotic transcription machinery. In the deduced structure of the complex TFIIF interacts with the polymerase in an extended conformation and almost completely eclipses the 4/7 sub-complex. The DNA being transcribed is bent at almost right angle with interaction of the TBP. The bent white arrow indicates the position of the transcription start site. The X-ray structure of the TBP-IIB-DNA complex was filtered to $15 \AA$ resolution for inclusion in the model (reprinted with permission from Asturias 2004). (B) With TBP at the core, the SAGA/TFIID complex is formed by several interacting proteins that are either common or unique to the respective complex. The coloured patches overlapping the TBP in the structure in A, represent the TBP associated factors making up the TFIID/SAGA. As described in the text, SAGA complex might replace the TFIID on the TATA containing promoters specifically upstream of the stress regulated genes. The TFIIF and the Rpb4/7 sub-complex of the polII are known to affect stress response and may affect interactions of the polymerase with downstream stress specific components. For more details, see text. 
complex component Spt3 has also shown to be important for induction of adequate response during nitrogen starvation (Laprade et al 2002). Though SAGA complex of eukaryotic transcriptional machinery does not have any protein factor which shows sequence similarities with $\sigma$ factor, several functions like ability to make contact with promoter DNA and with different components of transcriptional machinery for efficient and rapid induction of genes required during stress response, make SAGA complex a potential functional analogue of $\sigma^{\mathrm{s}}$.

3.1.2 TFIIF: This is a general transcription factor, initially identified by its association with RNA polymerase II and its requirement in transcription initiation. TFIIF is a heterotetramer complex composed of 2 large (TFIIF $\alpha /$ RAP74 in human and Tfg1 in $S$. cerevisiae) and two small (TFIIF $\beta /$ RAP30 in human and Tfg2 in $S$. cerevisiae) subunits (Flores et al 1990). Besides these two conserved subunits, $S$. cerevisiae also contains one smaller nonessential subunit, designated as Tfg3 (Henry et al 1992, 1994). Not only is Tfg3 present in TFIIF, it is also a part of other complexes like TFIID (thus also designated as Taf14) and SWI/SNF chromatin remodeling complexes (Cairns et al 1996). It has been reported that TFIIF complex is able to interact with TFIIA (Langelier et al 2001) and TFIIB (Ha et al 1993; Kimura and Ishihama 2004). Furthermore, genetic studies show that TFIIF functionally interacts with TFIIS, a general transcription factor required for efficient elongation by RNA pol II (Fish et al 2006).

There are several reasons which justify why TFIIF should be regarded functionally analogous to the $\sigma$ subunit of bacterial RNA polymerase. Firstly, both RAP74 and RAP30 show limited sequence homology with $\sigma^{70}$ (Garrett et al 1992; McCracken and Greenblatt 1991; Sopta et al 1989; Hamsey 1998). Secondly, Human TFIIF complex can bind to $E$. coli RNA polymerase and can be displaced by $\sigma^{70}$ (Hamsey 1998; McCracken and Greenblatt 1991). Thirdly, the distribution of Tfg2 in RNA pol II and TFIIF complex resembles the $\sigma$ factor distribution in bacterial holoenzyme as shown in figure $\mathrm{A}$ (Chung et al 2003). Furthermore, TFIIF is involved in recruitment of polymerase to form the PIC and for its stability, as shown in case of the $\sigma$ factor. All these features of TFIIF make it a potential $\sigma$ factor analog in eukaryotes. Recent studies using Tfg3 subunit of TFIIF in $S$. pombe have shown that $t f g 3^{-}$mutation is associated with stress sensitive phenotypes like temperature sensitivity, reduced cell growth during osmotic and heavy metal stress. Enhanced interaction of Tfg 3 with isolated TFIID during elevated temperature further supports role of this subunit of TFIIF under stress (Kimura and Ishihama 2004).

3.1.3 RPB4: Rpb4 is the fourth largest subunit of RNA polymerase II and is one of the two non essential subunits in
S. cerevisiae (Woychik and Young 1989) but Rpb4 homolog of $S$. pombe has been found to be essential for cell viability and is more similar in structure and function to those of higher eukaryotes than that of $S$. cerevisiae (Sakurai et al 1999). Several reports suggest that the absence of RPB4 leads to slow growth, temperature sensitivity and poor efficiency of survival during stationary phase (Woychik and Young 1989; Choder and Young 1993; Rosenheck and Choder 1998; Maillet et al 1999). It was later observed that cell wall integrity defects are also associated with rpb4 4 strain (Bourbonnais et al 2001). Furthermore, yeast cell lacking Rpb4 is defective in exhibition of two starvation specific phenotypes, e. g. sporulation and predisposition to forming pseudohyphal cells (Pillai et al 2003; Sampath et al 2003). Most of these phenotypic defects were involved in inability of the mutant cells to cope with the variety of stresses tested. Earlier work from our laboratory (Sharma and Sadhale 1999) had shown that the pseudohyphal phenotype of rpb4A cells was exaggerated when the levels of $\mathrm{Rpb} 7$, the interacting partner of $\mathrm{Rpb} 4$, were increased. Interestingly, homologs of the RPB7 gene from other eukaryotes showed different extent of pseudohyphal exaggeration indicating that the minor differences in the protein sequence might contribute to this phenotypic difference through protein-protein interactions (Khazak et al 1995; Singh et al 2004). Whole genome expression analysis done by our group to characterize this mutant showed that during permissive condition, this mutant affects only small subset of the genome, but in non permissive conditions like temperature stress, this mutant can affect differential expression of a large number of genes (Pillai et al 2003). In conclusion, these results suggest that Rpb4 of RNA polymerase II also acts during stress conditions similar to $\sigma^{\mathrm{s}}$ subunit of bacterial polymerase. Recent crystal structure of yeast RNA polymerase containing 12 subunits has suggested that the $\mathrm{Rpb} 4 / \mathrm{Rpb} 7$ sub-complex is present near the clamp region of the polymerase. In addition to this, this subcomplex is also closely associated with TFIIF (figure 3A). Though Rpb4 also does not share any sequence similarity with $\sigma$ subunit of bacterial polymerase, its several features discussed below, make it a worthy candidate to be called a functional analog of bacterial $\sigma$ subunit. Firstly, Rpb4 and its partner Rpb7, present in the polymerase, result in closed clamp conformation of RNA polymerase as seen in crystal structure of bacterial RNA polymerase holoenzyme containing core polymerase with $\sigma$ factor (Bushnell and Kornberg 2003; Armache et al 2003). Secondly, this subunit of polymerase has been speculated to play an important role in initiation by functioning as scaffold for further assembly of the components of PIC as shown in case of $\sigma$ factor. The Rpb4 protein also interacts with the CTD phosphatase Fcp1 (Kimura et al 2002) and is reported to be defective in transcriptional activation (Pillai et al 2001). Since CTD 
modification has direct bearing on the ability of polymerase to respond to transcription activation, Rpb4 in effect has an obvious role in modulating transcriptional activation crucial for stress responsive gene expression (Sampath and Sadhale 2005). Thus, for the first time a core subunit of the RNA polII has been shown to distinctly affect two specific stress responses like the stress sigma subunits of the prokaryotic cells.

\section{Concluding remarks}

It is observed that in nature living organisms encounter several stresses together. The mechanisms evolved in defense of these stresses indeed appear to be linked such that the organisms presented with one stress condition also show alacrity in responding to other apparently unrelated stresses. Mechanistically this has been achieved in prokaryotic systems by having promoter elements of several stress response genes being contacted by single stress sigma factor that determines the promoter selectivity of the transcription machinery. In eukaryotes since the responses to the variety of stresses are much more complex and varied the stress response regulatory system also has correspondingly increased in complexity. Strictly speaking sigma factor homologue does not exist in eukaryotes in that there is no single protein that allows the transcription machinery to be recruited at the promoter in eukaryotes. The components of the transcription machinery instead that are sufficient to recruit the polymerase at the promoter through DNA binding can be visualized as functionally analogous to sigma factors. The single protein sigma factor in the basal transcription machinery has been replaced by the multicomponent factors and is functionally best represented by the TFIID/TFIIB among the GTFs on the housekeeping genes while SAGA complex (in place of the TFIID), the TFIIF general transcription factor as well as the Rpb47 sub-complex of core subunit of the polII might play a significant role in the transcription of the stress regulated genes. All of these factors are conserved in evolution to a great extent and interestingly, they also are physically located in the transcription machinery in such a way as to be able to interact with each other (Figure 3B). That the stress response regulatory machinery should be close to the core of the transcription machinery to allow concerted co-regulation of genes involved in response to diverse stresses, is the theme that appears to be conserved in evolution.

\section{Acknowledgments}

Work in the PPS laboratory has been funded by grants from the Department of Biotechnology, Department of Science and Technology, Council for Scientific and Industrial
Research and Indian Council of Medical Research, New Delhi. We would like to thank Dr F J Asturias for allowing us to reproduce the structure representation of eukaryotic minimal transcription complex, originally published in his review in Current Opinion in Structural Biology (Asturias 2004). We like to thank members of the laboratory for support.

\section{References}

Armache K J, Kettenberger H and Cramer P 2003 Architecture of initiation-competent 12-subunit RNA polymerase II; Proc. Natl. Acad. Sci. USA 100 6964-6968

Asturias F J 2004 RNA polymerase II structure and organization of the pre-initiation complex; Curr. Opin. Struct. Biol. 14 121-129

Barbaric S, Reinke H and Horz W 2003 Multiple mechanistically distinct functions of SAGA at the PHO5 promoter; Mol. Cell. Biol. 23 3468-3476

Basehoar A D, Zanton S J and Pugh B F 2004 Identification and distinct regulation of yeast TATA box-containing genes; Cell 116 699-709

Bearson S M D, Benjamin W H J, Swords W E and Foster J W 1996 Acid induction of RpoS is mediated by the mouse virulence gene mviA of Salmonella typhimurium; J. Bacteriol. 178 2572-2579

Bhoite L T, Yu Y and Stillman D J 2001 The Swi5 activator recruits the Mediator complex to the HO promoter without RNA polymerase II; Genes Dev. 15 2457-2469

Bianchi A A and Barney F 1999 Hyperosmotic shock induces the ${ }^{32}$ and ${ }^{\mathrm{E}}$ stress regulons of Escherichia coli; Mol. Microbiol. 34 $1029-1038$

Bourbonnais Y, Faucher N, Pallotta D and Larouche C 2001 Multiple cellular processes affected by the absence of the Rpb4 subunit of RNA polymerase II contribute to the deficiency in the stress response of the yeast rpb4(delta) mutant; Mol. Gen. Genet. 264 763-772

Brand M, Yamamoto K, Staub A and Tora L 1999 Identification of TATA-binding protein-free TAFII-containing complex sub units suggests a role in nucleosome acetylation and signal transduction; J. Biol. Chem. 274 18285-18289

Brown C E, Howe L, Sousa K, Alley S C, Carrozza M J, Tan S, Workman J L 2001 Recruitment of HAT complexes by direct activator interactions with the ATM-related Tra1 subunit; Science 292 2333-2337

Bushnell D A and Kornberg R D 2003 Complete, 12-subunit RNA polymerase II at 4.1-A resolution: implications for the initiation of transcription; Proc. Natl. Acad. Sci. USA. 100 6969-6973

Cairns B R, Henry N L and Kornberg R D 1996 TFG3/TAF30/ ANC1, a component of the yeast SWI/SNF complex that is similar to the leukemogenic proteins ENL and AF-9; Mol. Cell. Biol. 16 3308-3316

Chadick J Z Asturias F J 2005 Structure of eukaryotic Mediator complexes; Trends Biochem. Sci. 30 264-271

Chang Y W, Howard S C and Herman P K 2004 The Ras/PKA signaling pathway directly targets the Srb9 protein, a component of the general RNA polymerase II transcription apparatus; Mol. Cell 15 107-116 
Choder M and Young R A 1993 A portion of RNA polymerase II molecules has a component essential for stress responses and stress survival; Mol. Cell Biol. 13 6984-6991

Chung W, Craighead J L, Chang L, Ezeokonkwo C, Bareket-Samish A, Kornberg R D and Asturias F J 2003 RNA Polymerase II/ TFIIF Structure and Conserved Organization of the Initiation Complex; Mol. Cell. 12 1003-1013

Delgado-Jarana J, Sousa S, Gonzalez F, Rey M, Llobell A 2006 ThHog1 controls the hyperosmotic stress response in Trichoderma harzianum; Microbiology 152 1687-1700

Dukan S and Nystrom T 1999 Oxidative stress defense and deterioration of growth-arrested Escherichia coli cells; J. Biol. Chem. 274 26027-26032

Fish R N, Ammerman M L, Davie J K, Lu B F, Pham C, Howe L, Ponticelli A S and Kane C M 2006 Genetic interactions between TFIIF and TFIIS; Genetics 173 1871-1884

Flores O, Ha I, and Reinberg D 1990 Factors involved in specific transcription by mammalian RNA polymerase II. Purification and subunit composition of transcription factor IIF; J. Biol. Chem. 265 5629-5634

Garrett K P, Serizawa H, Hanley J P, Bradsher J N, Tsuboi A, Arai N, Yokota T, Arai K, Conaway R C and Conaway J W 1992 The carboxyl terminus of RAP30 is similar in sequence to region 4 of bacterial sigma factors and is required for function; J. Biol. Chem. 267 23942-23949

Grossman A D, Erickson J W and Gross C A 1984 The htpR gene product of E. coli is a factor for heat-shock promoters; Cell $\mathbf{3 8}$ 383-390

Gruber T M and Gross C A 2003 Multiple sigma subunits and the partitioning of bacterial transcription space; Annu. Rev. Microbiol. 57 441-466

Ha I, Roberts S, Maldonado E, Sun X, Kim L U, Green M and Reinberg D 1993 Multiple functional domains of human transcription factor IIB: distinct interactions with two general transcription factors and RNA polymerase II; Genes Dev. 7 1021-1032

Hampsey M 1998 Molecular genetics of the RNA polymerase II general transcriptional machinery; Microbiol. Mol. Biol. Rev. $62465-503$

Henry, N L, Campbell A M, Feaver W J, Poon D, Weil P A and Kornberg R D 1994 TFIIF-TAF-RNA polymerase II connection; Genes Dev. 23 2868-2878

Henry N L, Sayre M H and Kornberg R D 1992 Purification and characterization of yeast RNA polymerase II general initiation factor g; J. Biol. Chem. 267 23388-23392

Holstege F C, Jennings E G, Wyrick J J, Lee T I, Hengartner C J, Green M R, Golub T R, Lander E S, and Young R A 1998 Dissecting the regulatory circuitry of a eukaryotic genome; Cell $95717-728$

Hoshijima M 2006 Mechanical stress-strain sensors embedded in cardiac cytoskeleton: $\mathrm{Z}$ disk, titin, and associated structures; Am. J. Physiol. Heart. Cvirc. Physiol. 290 H1313-H1325

Huisinga K L and Pugh B F 2004 A genome-wide housekeeping role for TFIID and a highly regulated stress-related role for SAGA in Saccharomyces cerevisiae; Mol. Cell. 13 573-585

Ishihama A 1990 Molecular assembly and functional modulation of Escherichia coli RNA polymerase; Adv. Biophys. 26 $19-31$
Khazak V, Sadhale P P, Woychik N A, Brent R and Golemis E A 1995 Human RNA polymerase II subunit hsRPB7 functions in yeast and influences stress survival and cell morphology; Mol. Biol. Cell. 6 759-775

Kimura M, Suzuki H and Ishihama A 2002 Formation of a carboxyterminal domain phosphatase (Fcp1)/TFIIF /RNA polymerase II (pol II) complex in Schizosaccharomyces pombe involves direct interaction between Fcp1 and the Rpb4 subunit of pol II; Mol. Cell Biol 22 1577-1588

Kimura M and Ishihama A 2004 Tfg3, a subunit of the general transcription factor TFIIF in Schizosaccharomyces pombe, functions under stress conditions; Nucleic Acids Res. 32 6706-6715

Langelier M F, Forget D, Rojas A, Porlier Y, Burton Z F and Coulombe B 2001 Structural and functional interactions of transcription factor (TF) IIA with TFIIE and TFIIF in transcription initiation by RNA polymerase II;. J. Biol. Chem. 276 38652-38657

Laprade L, Boyartchuk V L, Dietrich W F and Winston F 2002 Spt3 plays opposite roles in filamentous growth in Saccharomyces cerevisiae and Candida albicans and is required for C. albicans virulence; Genetics 161 509-519

Larschan E and Winston F 2001 The S. cerevisiae SAGA complex functions in vivo as a coactivator for transcriptional activation by Gal4; Genes Dev. 15 1946-1956

Lee Y C and Kim Y J 1998 Requirement for a functional interaction between mediator components Med6 and Srb4 in RNA polymerase II transcription; Mol. Cell. Biol. 18 5364-5370

Lee E, Karoonuthaisiri N, Kim H, Park J, Cha C, Kao C M and Roe J 2005 A master regulator B governs osmotic and oxidative response as well as differentiation via a network of sigma factors in Streptomyces coelicolor; Mol. Microbiol. 57 1252-1264

Lim, CY, Santoso, B, Boulay, T, Dong, E, Ohler, U, and Kadonaga, J T 2004 The MTE, a new core promoter element for transcription by RNA polymerase II; Genes Dev. 18 1606-1617

Maillet I, Buhler J M, Sentenac A and Labarre J 1999 Rpb4p is necessary for RNA polymerase II activity at high temperature; J. Biol. Chem. 274 22586-22590

Martinez E, Kundu T K, Fu J, and Roeder R G 1998 A human SPT3-TAFII31-GCN5-L acetylase complex distinct from transcription factor IID; J. Biol. Chem. 273 23781-23785

Martinez E, Palhan V B, Tjernberg A, Lymar E S, Gamper A M, Kundu T K, Chait B T and Roeder R G 2001 Human STAGA complex is a chromatin acety-lating transcription coactivator that interacts with pre-mRNA splicing and DNA damagebinding factors in vivo; Mol. Cell. Biol. 21 6782-6795

McCracken S, and Greenblatt J 1991 Related RNA polymerasebinding regions in human RAP30/74 and Escherichia coli sigma 70; Science 253 900-902

Muffler A, Traulsen D D, Lange R, and Hengge-Aronis R 1996 Posttranscriptional osmotic regulation of the $\sigma^{\mathrm{S}}$ subunit of RNA polymerase in Escherichia coli; J. Bacteriol. 178 1607-1613

Nonaka G, Blankschien M, Herman C, Gross C A and Rhodius V A 2006 Regulon and promoter analysis of the E. coli heat-shock factor, $\sigma^{32}$, reveals a multifaceted cellular response to heat stress; Genes Dev. 20 1776-1789

Ogryzko V V, Kotani T, Zhang X, Schlitz R L, Howard T, Yang X J, Howard B H, Qin J, and Nakatani Y 1998 Histone-liketAFs within the PCAF histone acetylase complex; Cell 94 35-44 
Pillai B, Sampath V, Sharma N and Sadhale P P 2001 Rpb4, a nonessential subunit of core RNA polymerase II of Saccharomyces cerevisiae is important for activated transcription of a subset of genes; J. Biol. Chem. 276 30641-30647

Pillai B, Verma J, Abraham A, Francis P, Kumar Y, Tatu U, Brahmachari S K and Sadhale P P 2003 Whole genome expression profiles of yeast RNA polymerase II core subunit, Rpb4, in stress and non stress conditions; J. Biol. Chem. 278 3339-3346

Rosenheck S, Choder M 1998 Rpb4, a subunit of RNA polymerase II, enables the enzyme to transcribe at temperature extremes in vitro; J. Bacteriol. 180 6187-6192

Sakurai H, Mitsuzawa H, Kimura M, Ishihama A 1999 The Rpb4 subunit of fission yeast Schizosaccharomyces pombe RNA polymerase II is essential for cell viability and similar in structure to the corresponding subunits of higher eukaryotes; Mol. Cell Biol. 19 7511-7518

Sampath V and Sadhale P 2005 Rpb4 and Rpb7: a sub-complex integral to multi-subunit RNA polymerases performs a multitude of functions; IUBMB Life. 57 93-102

Sampath V, Rekha N, Srinivasan N, Sadhale P 2003 The conserved and non-conserved regions of Rpb4 are involved in multiple phenotypes in Saccharomyces cerevisiae; J. Biol. Chem. 278 51566-51576

Santos B C, Chevaile A, Kojima R and Gullans S R 1998 Characterization of the Hsp110/SSE gene family response to hyperosmolality and other stresses; Am. J. Physiol. Renal. Physiol. 274 F1054-F1061

Schaik W and Abee T 2005 The role of B in the stress response of Gram-positive bacteria - targets for food preservation and safety; Curr. Opin. Biotech. 16 218-224

Shao H, Revach M, Moshonov S, Tzuman Y, Gazit K, Albeck S, Unger T, and Dikstein R 2005 Core promoter binding by histone-like TAF complexes; Mol. Cell Biol. 25 206-219

Sharma N and Sadhale P P 1999 Overexpression of the gene for Rpb7 subunit of yeast RNA polymerase II rescues the phenotypes associated with the absence of the largest, nonessential subunit Rpb4; J. Genet. 78 149-156

Singh S R, Rekha N, Pillai B, Singh V, Naorem A, Sampath V, Srinivasan N, and Sadhale P P 2004 Domainal organization of the lower eukaryotic homologs of the yeast RNA polymerase II core subunit Rpb7 reflects functional conservation; Nucleic Acids Res. 32 201-210

Sopta M, Burton Z F and Greenblatt J 1989 Structure and associated DNA-helicase activity of a general transcription initiation factor that binds to RNA polymerase II; Nature (London) 341 410-414

Thomas M C and Chiang C M 2006 The general transcription machinery and general cofactors; Crit. Rev. Biochem. Mol. Biol. 41 105-178

Vassylyev D G, Sekine S, Laptenko O, Lee J, Vassylyeva M N, Borukhov S and Yokoyama S 2002 Crystal structure of a bacterial RNA polymerase holoenzyme at 2.6 A resolution; Nature (London) 417 712-719

Wecke T, Veith B, Ehrenreich A and Mascher T 2006 Cell Envelope Stress Response in Bacillus licheniformis: Integrating Comparative Genomics, Transcriptional Profiling, and Regulon Mining To Decipher a Complex Regulatory Network; $J$. Bacteriol. 188 7500-7511

Woychik N A and Young R A 1989 RNA polymerase II subunit RPB4 is essential for high- and low-temperature yeast cell growth; Mol. Cell. Biol. 9 2854-2859

Zanton S J and Pugh B F. 2004. Changes in genomewide occupancy of core transcriptional regulators during heat stress; Proc. Natl. Acad. Sci. USA 101 16843-16848

ePublication: 29 March 2007 